\title{
Label-Free SERS Detection of Bovine Serum Albumin Protein Based on Au@Ag Core/Shell Nanobricks
}

\section{Huang Liu, Yi Chen*}

State Key Laboratory of Bioelectronics, Jiangsu Key Laboratory for Biomaterials and Devices, School of Biological Science \& Medical Engineering, Southeast University, Nanjing, China

\author{
Email address: \\ liuh93@qq.com (Huang Liu), yichen@seu.edu.cn(Yi Chen) \\ ${ }^{*}$ Corresponding author
}

\section{To cite this article:}

Huang Liu, Yi Chen. Label-Free SERS Detection of Bovine Serum Albumin Protein Based on Au@Ag Core/Shell Nanobricks. Science Discovery. Vol. 7, No. 3, 2019, pp. 147-151. doi: 10.11648/j.sd.20190703.13

Received: April 19, 2019; Accepted: May 23, 2019; Published: June 15, 2019

\begin{abstract}
In this study, a simple yet effective protein detection method was established by using bimetallic Au@Ag core-shell nanobrick as SERS active building blocks. The Au@Ag core-shell nanobrick is synthesized by well-defined 'two-step' growing method, enabling uniform and controllable silver shell growth on the surface of the gold nanorods induced by surfactant replacement. The thickness of the silver shell can be programmably adjusted by controlling the amount of silver nitrate in the system, which in turn realized the controllable adjustment of the optical properties of Au@Ag core-shell nanobrick. We selected bovine serum albumin as a model protein and established a highly-sensitive assay for label-free SERS detection of protein. The detection limit can reach to as low as $0.5 \mu \mathrm{g} / \mathrm{mL}$, enabling promising avenue for its clinical application in ultrasensitive biomarker detection.
\end{abstract}

Keywords: Plasmonic Nanoparticle, Au@Ag Core-shell Nanobrick, SERS, Label-Free Protein Detection

\section{基于金银核壳纳米砖的无标记法SERS检测牛血清白蛋白}

刘晃, 陈怡

生物电子学国家重点实验室, 江苏省生物材料与器件重点实验室, 东南大学生物科学与医学工程学院, 南京, 中国

邮箱

liuh93@qq.com(刘晃), yichen@seu.edu.cn(陈怡)

摘要: 本研究以新型双金属金银核壳纳米砖作为SERS活性基元, 开发了一种简单高效的蛋白质分子痕量检测方法。通 过可控的两步合成方法, 实现了基于表面活性剂置换和引导的金银核壳纳米砖结构生长和形貌调控, 同时通过体系中 硝酸银的浓度调控来实现银壳层的厚度, 从而整体达到金银核壳纳米砖光学性能的可控设计与调节。我们选择牛血清 白蛋白作为模式蛋白, 建立了以牛血清白蛋白为检测靶标的无标记SERS高灵敏检测方法, 其检测限度约为 $0.5 \mu \mathrm{g} / \mathrm{mL}$, 为后续工作中实现蛋白的特异性捕获、富集及高灵敏无创检测提供了应用基础。

关键词: 贵金属纳米粒子, 金银核壳纳米砖, 表面增强拉曼散射, 无标记蛋白检测

\section{1. 引言}

最近，表面增强拉曼散射（SERS）作为一种检测手 段, 由于具有的高灵敏度和选择性、良好的稳定性等优势,
已成为生物化学、生物医学、食品安全和环境监测领域应 用最广泛的分析技术之一 $[1,2]$ 。近年来, SERS在生物领 域 (例如蛋白质 [3], DNA / RNA[4], 细胞和细菌的研究) 的应用越来越引起研究人员的关注。基于 SERS的生物分 
子检测 [5]主要有两种方法, 无标记法 [6]和外源SERS标记 法[7]。无标记法旨在在没有拉曼标签的情况下直接获得生 物分子的SERS光谱; 外源SERS标记方法使用拉曼标记来 间接地检测生物分子。相比较而言在检测生物分子方面更 加直接可靠的方法, 并且它比外在SERS标记方法更方便。 但是, 在某些情况下, 无标记检测也具有一些局限性: (1) 它的灵敏度有限, 因为相对低浓度的生物分子的固有 SERS光谱通常难以检测; (2) 它在一些复杂的混合物中 表现出较差的选择性, 因为重叠的拉曼谱带使样品识别变 得困难。尽管存在这些局限性, 近年来, 在开发基于SERS 的无标记蛋白质检测方法方面进行了大量研究 [7-9]。Zhao, Ozaki和他们的同事进行了系统的工作 [10], 以提高基于该 方法的可靠性和多功能性, 表明基于SERS的无标记法的 高灵敏度、选择性检测生物分子是非常具有潜力的。

金纳米粒子 [11], 尤其是金纳米棒 [12], 由于其化学 稳定性、在可见光和近红外光谱区域内具有可调谐的等离 子共振谱带等性能, 已被广泛用作分析和生物化学应用等 领域 [13]。然而单组分金纳米粒子有时难以满足具体应用 的需要, 就需要两到三种的贵金属组合的纳米粒子 $[14,15]$ 。 与其他贵金属相比, 银纳米粒子具有更强的拉曼增强效果, 除此之外银纳米粒子还表现出比金纳米粒子更高的折射 率灵敏度和更大的太阳能转换效率。然而, 具有均匀形状 和窄尺寸分布的各向异性银纳米粒子的制备仍具有挑战 性。金纳米棒由于其可调节的局域表面等离子共振 (LSPR) 性能使得其获得了广泛的应用, 以金纳米棒为核的核壳型 金属纳米结构的研究引起了人们的极大兴趣, 到目前为止, 多种不同形貌的核壳型金属纳米结构陆续被报道出来。与 其他贵金属相比, 金银核壳纳米棒是研究的最多的双金属 纳米结构, 通过控制不同的生长条件, 例如 $\mathrm{pH}$ 值、表面活 性剂和温度等条件可以获得许多不同的形状, 例如圆柱体, 哑铃型, 长方体和八面体等[16-21]。

在本研究中, 我们旨在用新型双金属金银核壳纳米砖 作为SERS活性基元开发一种蛋白质检测和鉴定方法, 采 用双金属金银核壳纳米砖-蛋白复合体的形式通过SERS检 测实现对蛋白的痕量检测。我们使用无标记法常用的模 式蛋白牛血清白蛋白测试该方法的性能。这种方法表现出 的高灵敏度为后续工作中实现蛋白的特异性高灵敏无创 检测提供了应用基础。

\section{2. 实验部分}

\section{1. 材料与仪器}

四氯金酸三水合物 $\left(\mathrm{HAuCl}_{4} \cdot 3 \mathrm{H}_{2} \mathrm{O}, \geq 49.0 \% \mathrm{Au}\right.$ basis $)$,

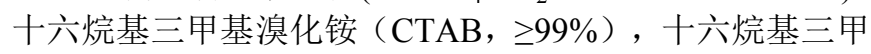
基氯化铵（CTAC，25 wt.\% in $\mathrm{H}_{2} \mathrm{O}$ ), L-抗坏血酸 ( $\mathrm{AA}$, $\geq 99 \%$ ），硼氢化钠( $\mathrm{NaBH} 4 ， \geq 99 \%)$ 购自 Sigma-Alorich; 硝酸银 $\left(\mathrm{AgNO}_{3}, 99.99 \%\right)$ 购自Aladdin; 牛血清白蛋白 (BSA, Fraction V) 购自 Solarbio; 实验用水均为超纯水, 采自Milli-Q Reference系统。

用日本SHIMADZU公司的紫外-可见分光光度计 (UV-2450)表征金纳米棒和金银核壳纳米砖的光学性质, 用美国FEI公司的扫描电子显微镜 (SEM, Quanta 250 FEG)
和日本HITACHI公司的透射电子显微镜 (TEM, HT7700) 观测金纳米棒的表面形貌, 用美国Thermo公司的激光显微 共聚焦拉曼光谱仪（DXR 2Xi）来对其进行SERS检测。

\section{2. 实验步骤}

\subsection{1. 金种子的制备}

取 $5 \mathrm{~mL} 0.2 \mathrm{M} \mathrm{CTAB}$ 水溶液于烧杯中, 加入 $5 \mathrm{~mL} 1$ $\mathrm{mM}$ 的 $\mathrm{HAuCl}_{4}$ 溶液, 充分混合后快速加入 $0.6 \mathrm{~mL}$ 冰水配置 的 $10 \mathrm{mM} \mathrm{NaBH}$ 水溶液, 溶液由浅黄色变为棕黄色., 快 速搅拌2 min后置于室温静置1 h 后备用。

\subsection{2. 金纳米棒的制备}

取 $5 \mathrm{~mL} 0.2 \mathrm{M} \mathrm{CTAB}$ 水溶液于 $15 \mathrm{~mL}$ 离心管中, 加入 5 $\mathrm{mL} 1 \mathrm{mM} \mathrm{HAuCl}_{4}$ 水溶液、 $0.2 \mathrm{~mL} 4 \mathrm{mM} \mathrm{AgNO}$ 水溶液, 上下颠倒几次使之充分混合, 然后加入 $0.08 \mathrm{~mL} 80 \mathrm{mM}$ $\mathrm{AA}$ 水溶液, 上下颠倒几次溶液随之从淡黄色变为无色, 加入 $0.012 \mathrm{~mL}$ 晶种溶液, 充分混合后置于 $30^{\circ} \mathrm{C}$ 水浴静置生 长 $2 \mathrm{~h}$ 。

\subsection{3. 金银核壳纳米砖的制备}

将上述合成的金纳米棒取 $10 \mathrm{~mL}$ 以转速7000 rpm离心 $20 \mathrm{~min}$, 去除上清液, 加入等量的 $80 \mathrm{mM} \mathrm{CTAC}$ 水溶液重 悬, 该步骤重复两次以确保表面活性剂的充分置换; 将上 述置换后的金纳米棒置于 $20 \mathrm{~mL}$ 的玻璃瓶中, 顺序加入 0.8 $\mathrm{mL}$ 的 $10 \mathrm{mM} \mathrm{AgNO}$ 水溶液, $0.4 \mathrm{~mL}$ 的 $0.1 \mathrm{M} \mathrm{AA}$ 水溶液, 充分混匀后置于 $60^{\circ} \mathrm{C}$ 水浴中速摚拌反应 $3 \mathrm{~h}$; 反应完成后, 撤去水浴, 待其冷却至室温后以转速7000 rpm离心 $20 \mathrm{~min}$, 去除上清液加入等量去离子水重悬。

\subsubsection{BSA蛋白与金银核壳纳米砖复合物的制备}

将上述制备的金银核壳纳米砖浓缩 $10 \times$, 与等体积的 不同浓度的BSA $(1 \mathrm{mg} / \mathrm{mL} 、 0.1 \mathrm{mg} / \mathrm{mL} 、 0.01 \mathrm{mg} / \mathrm{mL} 、 1$ $\mu \mathrm{g} / \mathrm{mL}$ ）水溶液混合（BSA终浓度分别为 $500 \mu \mathrm{g} / \mathrm{mL} 、 50$ $\mu \mathrm{g} / \mathrm{mL} 、 5 \mu \mathrm{g} / \mathrm{mL} 、 0.5 \mu \mathrm{g} / \mathrm{mL}$ ), 室温下静置 $1 \mathrm{~h}$, 然后将 金银核壳纳米砖-BSA蛋白复合物置于 $1 \times 1 \mathrm{~cm}^{2}$ 硅片表面上 待其干燥后用于SERS表征。测试所用的仪器为 Thermo Scientific DXR 2Xi激光显微共聚焦拉曼光谱仪, 选择633 $\mathrm{nm}$ 的二极管激光器作为光源, 选择目镜为 $50 \times$, 所有测量 均使用 $2 \mathrm{~mW}$ 的激光功率, 激光在样品上的曝光时间均为 $10 \mathrm{~S}$ 。每个样品均测量五次以上。

\section{3. 实验结果与讨论}

\section{1. 金纳米棒的表征}

金纳米棒具有两个等离激元振动模式[22], 一个是电 子的横向等离激元共振产生的横向吸收峰 (TSPW), 另 一个是电子的纵向等离激元共振产生的纵向吸收峰 (LSPW)。如图1 (A) 所示, 制备的金纳米棒的 TSPW 在 $520 \mathrm{~nm}$ 处附近, 其LSPR在 $660 \mathrm{~nm}$ 处附近, 峰型及其半 峰宽均良好, 无其他杂峰出现。金纳米棒的TEM图如图1

(B) 所示, 可以看出金纳米棒单分散性较为良好, 基本 没有其他球状杂质。 


\section{2. 金银核壳纳米砖的表征}

金银核壳纳米砖的紫外可见吸收光谱图和其TEM图 如图2所示。如图2（A）所示，金银核壳纳米砖表现出四 个等离激元共振带[19], 将等离激元谱带从低能量到高能 量依次定义为峰 1-4。峰1和峰2分别代表纵向吸收峰和横

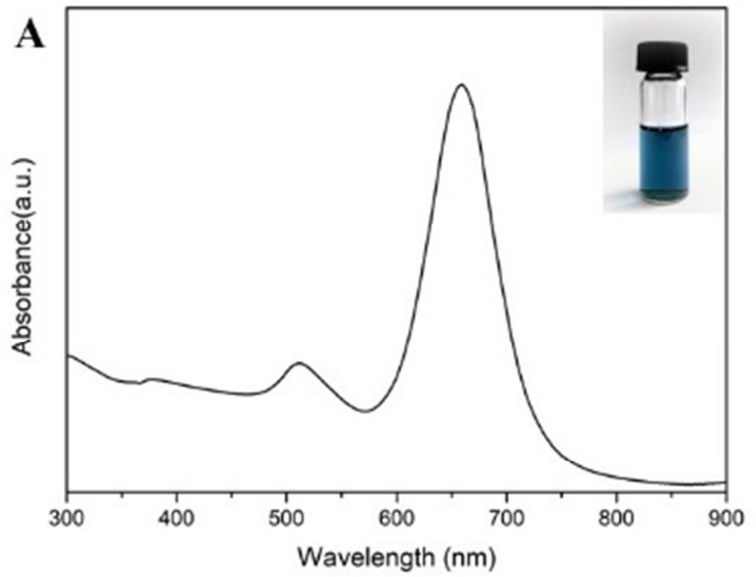

向吸收峰, 当银壳的厚度达到一定值之后出现峰 3 和 4 , 其 具体的振动模式尚不明确。金银核壳纳米砖表现出胶体的 性质, 透光呈现出橙色或橙红色。金银核壳纳米砖的TEM 图如图2（B）所示, 其外观形貌表现为长方体结构, 棱角 较为尖锐, 其中金纳米棒侧边的银壳厚度比两端的银壳要 厚。通过调整体系中 $\mathrm{AgNO}_{3}$ 的量可以调整银壳的厚度。

图1 金纳米棒的紫外可见吸收光谱图（A）和TEM图（B）。

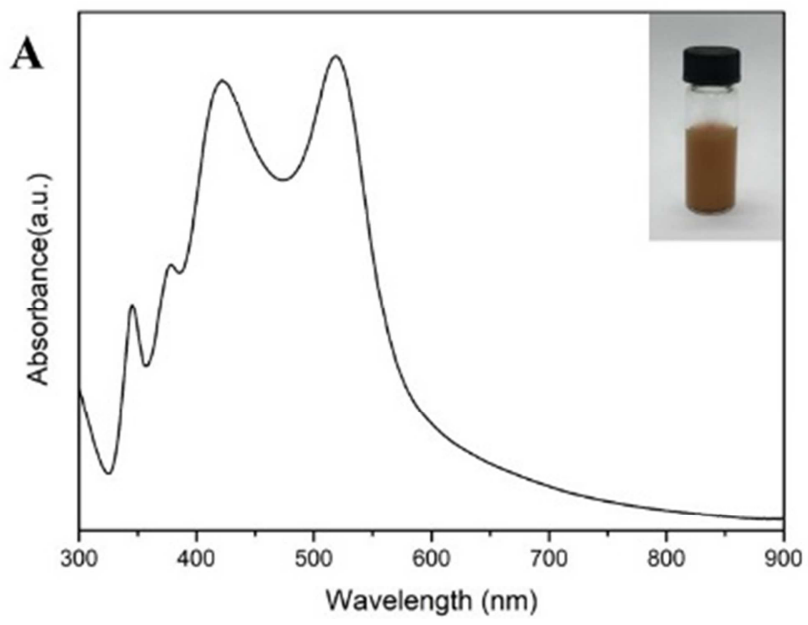

图2 金银核壳纳米砖的紫外可见吸收光谱图（A）和TEM图（B）。

\section{3. 基于金银核壳纳米砖的BSA检测}

本研究所采用SERS检测蛋白质的方法为无标记法。 无标记法充分体现了拉曼光谱的优势, 因为它能提供待检 测生物分子的固有振动特性。所用的待测蛋白质为牛血清 白蛋白（BSA） [23], BSA常作为SERS法检测蛋白的模式 蛋白。BSA是一种球状水溶性蛋白质, 其主要结构是已知 的, 分子量为 $66267 \mathrm{Da}$, 等电点为 $\mathrm{pH} 4.5-5.0$, 这意味着在 中性溶液中, BSA整个分子带负电荷。然而, BSA具有带 负电荷的氨基酸结构域, 如谷氨酸或天冬氨酸, 以及带正 电的结构域, 如赖氨酸或组氨酸。因此, BSA可以在中性 环境中吸附在带正电荷和带负电的粒子表面上。纯BSA固 体粉末的拉曼图谱如图3所示。纯BSA的SERS峰出现在 640、756、847、887、1000、1029、1204、1441和 $1666 \mathrm{~cm}^{-1}$
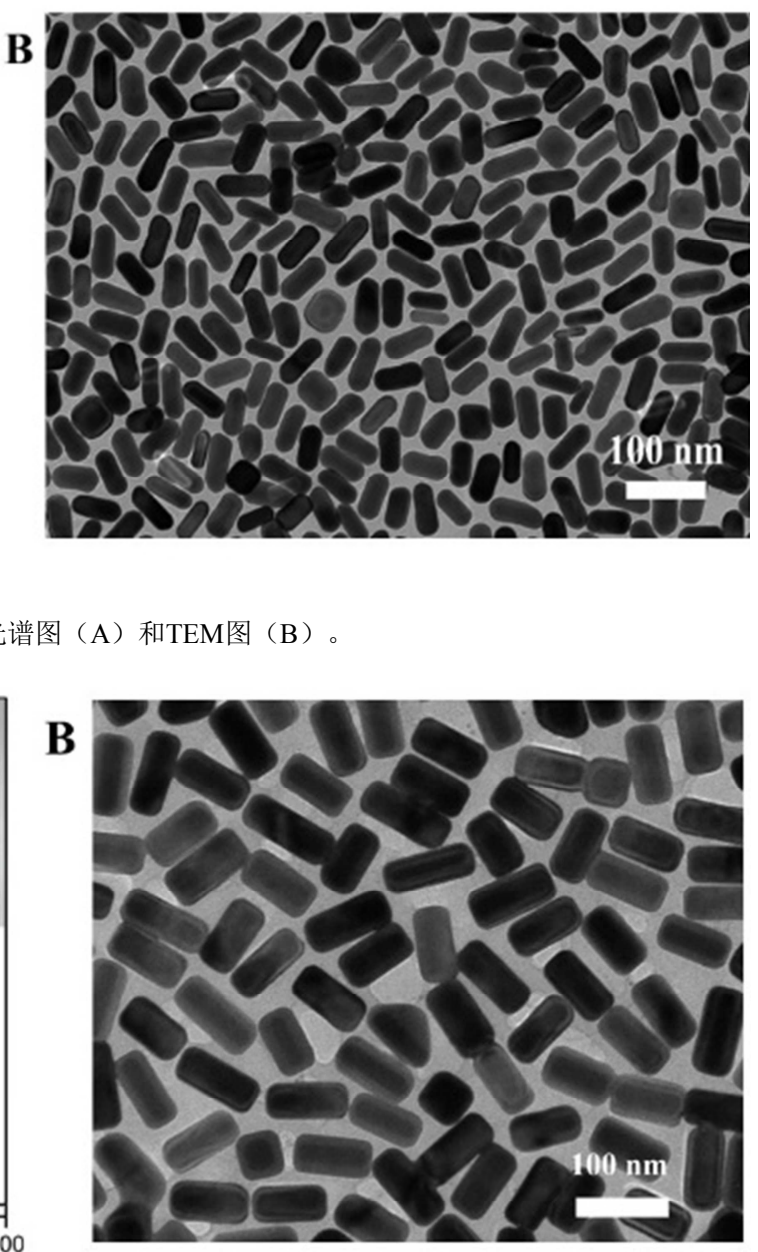

等位置, 其中较强的 $1000 \mathrm{~cm}^{-1}$ 附近位置的特征峰是由苯丙 氨酸（Phe）的苯环振动引起的 [24]。

如上文中所提到的, BSA具有带正电和带负点的结构 域使得其可以通过静电吸附结合到金银核壳纳米砖表面。 其次由于BSA中的半胱氨酸（cys）残基含有的颈基也可 以通过Ag-S键与金银核壳纳米砖以共价键方式结合。当 BSA浓度较高时（ $\geq 50 \mu \mathrm{g} / \mathrm{mL} ）$ 时, BSA取代掉大部分金 银核壳纳米砖表面的 CTAC, 会使得金银核壳纳米砖因缺 少表面稳定剂而聚沉, 其SEM图如图4 (B) 所示。对金银 核壳纳米砖与BSA蛋白的复合物进行SERS检测, 其SERS 图谱如图4 (A) 所示。由于该方法检测蛋白会使得蛋白的 变性, 使得其具有与原状态不同的拉曼特征峰。我们重点 比较其在 $1000 \mathrm{~cm}^{-1}$ 处附近的特征峰, 该位置的特征峰是由 BSA所具有的色氨酸 (Trp) 和苯丙氨酸 (Phe) 的振动引 起的。通过比较该特征峰的强度, 可以看出随着BSA浓度 
的逐渐降低, 该特征峰的强度也呈现出逐渐递减的趋势。 当BSA浓度减少至 $0.5 \mu \mathrm{g} / \mathrm{mL}$ 时, 该特征峰的强度已经非常 弱, 表明该方法检测BSA的限度能达到 $8 \mathrm{nM}$ 。

基于SERS的无标记法蛋白质检测的三个主要问题是 灵敏度、重现性和天然状态。灵敏度主要是由SERS基底 所决定的。第二个也是最主要的挑战是重复性差, 特别是 对于没有发色团的蛋白质。第三个问题是蛋白质的变质问 题。通过本文实验可知, 通过采用金银核壳纳米砖与BSA 蛋白结合进行SERS检测可以实现待测蛋白较高限度的 SERS增强效果; 其次, 上述各浓度BSA的实验数据均是 通过重复测量多个位置, 显示出极高的重复性, 说明使得 使用该金银核壳纳米砖-BSA复合物检测蛋白质具有较好 的重复性。基于该方法的蛋白检测仍旧会造成蛋白一定程 度的变性, 但通过检测氨基酸残基可以一定程度的弥补蛋 白变性带来的局限性。

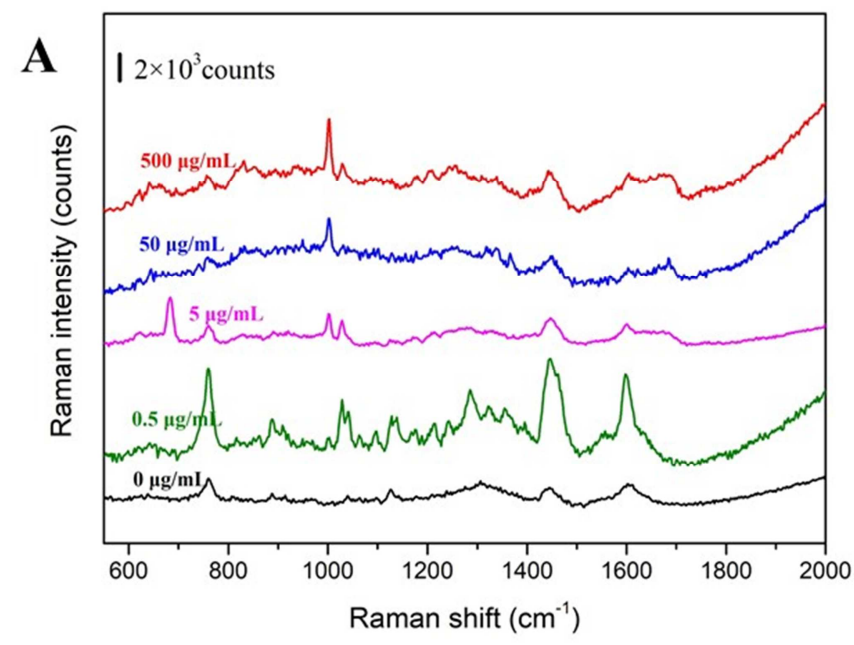

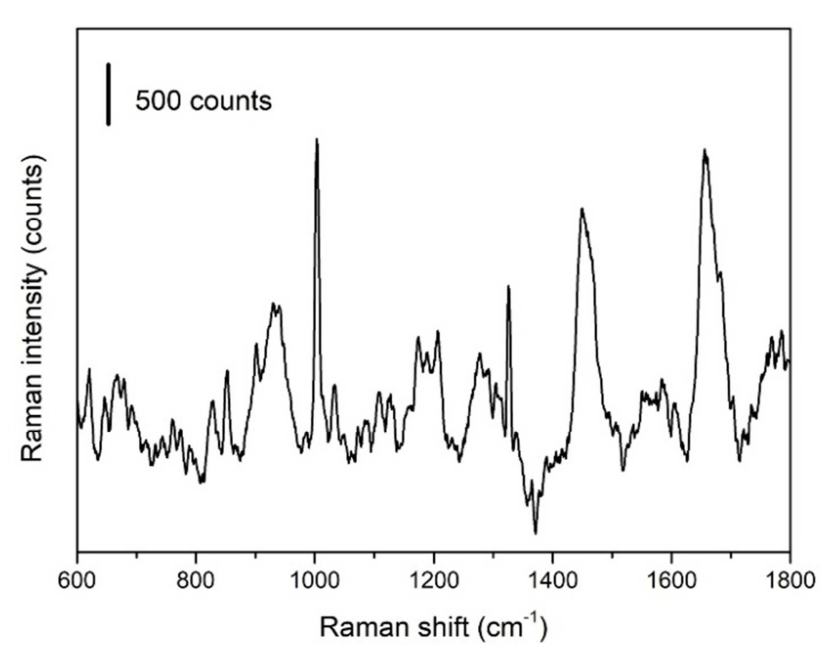

图3 BSA固体粉末的拉曼图。

$\mathbf{B}$

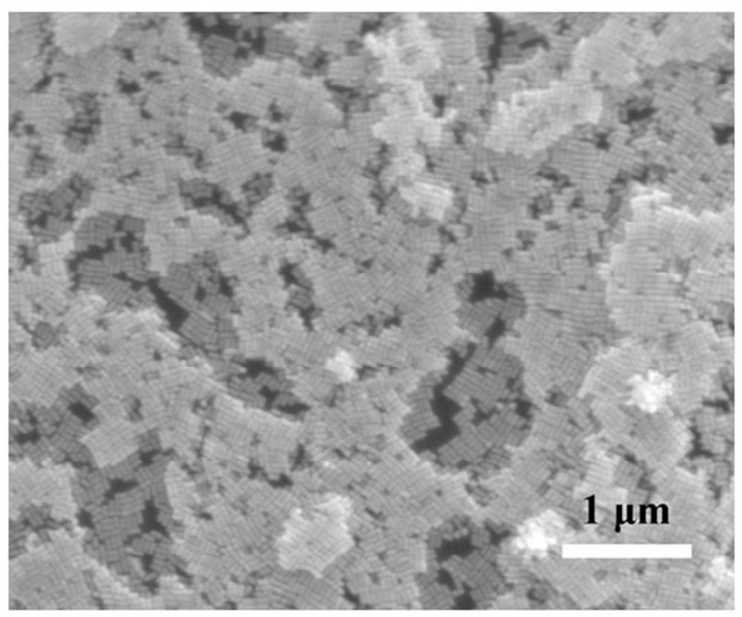

图4 金银核壳纳米砖-BSA复合物的SEM图(A)和金银核壳纳米砖-BSA蛋白复合物的SERS图谱(B), BSA浓度分别为 $500 \mu \mathrm{g} / \mathrm{mL} 、 50 \mu \mathrm{g} / \mathrm{mL} 、 5 \mu \mathrm{g} / \mathrm{mL}$ 、 $0.5 \mu \mathrm{g} / \mathrm{mL} 、 0 \mu \mathrm{g} / \mathrm{mL}$ 。

\section{4. 结论}

为了解决蛋白质SERS检测中重现性差，检测灵敏度 低的问题，我们提出了一种基于新型双金属金银核壳纳米 砖作为SERS活性激元用于蛋白质的无标记法SERS检测。 通过控制双金属金银核壳纳米砖银壳的厚度可以实现在 可见光到近红外光谱区域中可控调整其等离激元波长, 以 提供高度增强的电磁场实现其作为SERS活性激元的性能。 我们通过以BSA作为目的蛋白, 初步实验结果表明该方法 对BSA的检测限度能达到 $0.5 \mu \mathrm{g} / \mathrm{mL}$, 表明通过该方法可以 实现高度可重复和较强的SERS信号。实验结果证实了该 方法简单有效, 并且可以显着提高无标记SERS检测方法 的可靠性、再现性和灵敏度。

\section{致谢}

感谢国家自然科学基金（NO. 21501027），中国科协 青年人才托举工程（NO.YESS20150050），江苏省“双创
计划”, 苏州市应用基础研究项目（SYG201528），中央 高校基本科研业务费专项资金对本研究的资助。

\section{参考文献}

[1] Sharma B, Frontiera R R, Henry A-I, et al. SERS: Materials, applications, and the future [J]. Mater Today, 2012, 15(1-2): 16-25.

[2] Jia M, Li S, Zang L, et al. Analysis of Biomolecules Based on the Surface Enhanced Raman Spectroscopy [J]. Nanomaterials (Basel), 2018, 8(9):

[3] Singhal K, Kalkan A K. Surface-enhanced Raman scattering captures conformational changes of single photoactive yellow protein molecules under photoexcitation [J]. J Am Chem Soc, 2009, 132(2): 429-431.

[4] Barhoumi A, Zhang D, Tam F, et al. Surface-enhanced Raman spectroscopy of DNA [J]. J Am Chem Soc, 2008, 130(16): 5523-5529. 
[5] 陈雷, 孔卫贺, 韩晓霞, 等. 表面增强拉曼光谱(SERS)技术 对非标记蛋白质的研究进展 [J]. 光谱学与光谱分析, 2016, 36(10): 3087-3091.

[6] Ray S, Mehta G, Srivastava S. Label-free detection techniques for protein microarrays: prospects, merits and challenges [J]. Proteomics, 2010, 10(4): 731-748.

[7] Xu L J, Zong C, Zheng X S, et al. Label-free detection of native proteins by surface-enhanced Raman spectroscopy using iodide-modified nanoparticles [J]. Anal Chem, 2014, 86(4): 2238-2245.

[8] Kahraman M, Wachsmann-Hogiu S. Label-free and direct protein detection on $3 \mathrm{D}$ plasmonic nanovoid structures using surface-enhanced Raman scattering [J]. Anal Chim Acta, 2015, 856(1): 74-81.

[9] Cao X, Wang Z, Bi L, et al. Label-Free Detection of Human Serum Using Surface-Enhanced Raman Spectroscopy Based on Highly Branched Gold Nanoparticle Substrates for Discrimination of Non-Small Cell Lung Cancer [J]. Journal of Chemistry, 2018, 2018(1): 1-13.

[10] Han X X, Huang G G, Zhao B, et al. Label-free highly sensitive detection of proteins in aqueous solutions using surface-enhanced Raman scattering [J]. Anal Chem, 2009, 81(9): 3329-3333.

[11] Klekotko M, Matczyszyn K, Siednienko J, et al. Bio-mediated synthesis, characterization and cytotoxicity of gold nanoparticles [J]. Phys Chem Chem Phys, 2015, 17(43): 29014-29019.

[12] Marangoni V S, Cancino-Bernardi J, Zucolotto V. Synthesis, physico-chemical properties, and biomedical applications of gold nanorods - a review [J]. Journal of biomedical nanotechnology, 2016, 12(6): 1136-1158.

[13] Chen Y, Fu J, Ng K C, et al. Free-Standing PolymerNanoparticle Superlattice Sheets Self-Assembled at the AirLiquid Interface [J]. Crystal Growth \& Design, 2011, 11(11): 4742-4746.

[14] Chen N T, Tang K C, Chung M F, et al. Enhanced plasmonic resonance energy transfer in mesoporous silica-encased gold nanorod for two-photon-activated photodynamic therapy $[\mathrm{J}]$. Theranostics, 2014, 4(8): 798-807.
[15] Bai Y, Gao C, Yin Y. Fully alloyed $\mathrm{Ag} / \mathrm{Au}$ nanorods with tunable surface plasmon resonance and high chemical stability [J]. Nanoscale, 2017, 9(39): 14875-14880.

[16] Xiang Y, Wu X, Liu D, et al. Gold nanorod-seeded growth of silver nanostructures: from homogeneous coating to anisotropic coating [J]. Langmuir, 2008, 24(7): 3465-3470.

[17] Park K, Drummy L F, Vaia R A. Ag shell morphology on Au nanorod core: role of $\mathrm{Ag}$ precursor complex [J]. J Mater Chem, 2011, 21(39): 15608-15618.

[18] Cho E C, Camargo P H, Xia Y. Synthesis and characterization of noble-metal nanostructures containing gold nanorods in the center [J]. Adv Mater, 2010, 22(6): 744-748.

[19] Okuno Y, Nishioka K, Kiya A, et al. Uniform and controllable preparation of $\mathrm{Au}-\mathrm{Ag}$ core-shell nanorods using anisotropic silver shell formation on gold nanorods [J]. Nanoscale, 2010, 2(8): 1489-1493.

[20] Chen Y, Si K J, Sikdar D, et al. Ultrathin Plasmene Nanosheets as Soft and Surface-Attachable SERS Substrates with High Signal Uniformity [J]. Adv Opt Mater, 2015, 3(7): 919-924.

[21] Khlebtsov B N, Liu Z, Ye J, et al. Au@Ag core/shell cuboids and dumbbells: Optical properties and SERS response [J]. J Quant Spectrosc Radiat Transfer, 2015, 167(1): 64-75.

[22] Chen H, Shao L, Li Q, et al. Gold nanorods and their plasmonic properties [J]. Chem Soc Rev, 2013, 42(7): 2679-2724.

[23] Liu N, Chi Y, Dong L, et al. Label-Free Detection of Bovine Serum Albumin Protein Based on $\mathrm{SiO}_{2} / \mathrm{Au}$ Nanoshells as Near-Infrared Surface-Enhanced Raman Spectroscopy Nanoprobe [J]. J Nanosci Nanotechnol, 2016, 16(7): 7103-7109.

[24] Shao M, Lu L, Wang H, et al. Microfabrication of a new sensor based on silver and silicon nanomaterials, and its application to the enrichment and detection of bovine serum albumin via surface-enhanced Raman scattering [J]. Microchimica Acta, 2008, 164(1-2): 157-160. 